\title{
The use of doppler ultrasound in evaluation and follow-up of arteriovenous fistula patients
}

\author{
Nail Kahraman, Kadir Kaan Özsin \\ Department of Cardiovascular Surgery, University of Health Sciences, Bursa Yüksek İhtisas Training and Research Hospital, Bursa, \\ Turkey
}

DOI: 10.18621 eurj.376240

\begin{abstract}
Objectives: The The aim of the present study was to search the effect of preoperative Doppler ultrasonography (DUS) of the concerning limb on AVF patency for arteriovenous fistula (AVF) to be performed on the patients with end-stage renal disease.

Methods. One hundred and three patients were enrolled into the study. The exclusion criteria were previous central catheter procedure, history of thrombophlebitis on the upper limb and previous surgery on the upper limb. Among the remaining patients, those who fulfilled the physical examination criteria were included. The patients were divided into two groups as the control, DUS (-) group and the study group, DUS (+). The patients in the control group were taken into the procedure after a physical examination only. Brescio-Cimino method was preferred for all patients. Function of the AVF was controlled on the procedure day, at day 10, months 1, 3 and 6 as well as year 1 after the procedure. The results in both groups were statistically evaluated.

Results: Twenty patients in the DUS (+) group (50\% male, mean age: $57.25 \pm 13.34$ years) and 20 patients in the DUS (-) group (45\% male, mean age: $56.10 \pm 12.35)$ were recorded in the study. Cumulative primary patency rates between DUS (+) group and DUS(-) group for 12 months were $95 \%$ and $65 \%$, respectively (logrank, $p=0.022$ ).

Conclusion: We believe that the DUS performed before AVF procedure would increase the primary patency rates of AVF created between the most convenient vessels and reduce the procedure failure.
\end{abstract}

Keywords: Hemodialysis, arteriovenous fistula, doppler ultrasonography, primary patency

Received: January 8, 2018; Accepted: February 19, 2018; Published Online: March 15, 2018

$\mathrm{T}$ he patient population with end-stage renal disease (ESRF) consistently increases worldwide. The number of the patients who need dialysis treatment increases about $10 \%$ of current patients every year [1]. Arteriovenous fistula (AVF) procedures are the most common methods implemented to facilitate the hemodialysis process and increase the life standards of the patient [2]. The blood flow required for hemodialysis depends on the venous blood flow of the arteriovenous anastomosis to be created. The success of the procedure is dependent to the anastomosis technique and the formation of the vessel selected for surgical procedure. The AVFs were first suggested by Cimino-Brescia in 1966; although such technique was modified by technical developments up to date, the target always remained the same [3].

Doppler Ultrasonography (DUS) is the most applicable and complication-free diagnostic method. Other advantages of this method include not

Address for correspondence: Nail Kahraman, MD., University of Health Sciences, Bursa Yüksek İhtisas Training and Research Hospital, Bursa, Turkey E-mail: nailkahraman1979@gmail.com,Fax:+902242955497 
administration of any contrast agent, availability and dynamic approach. Vascular system mapping may be performed before the procedure. It was shown that unnecessary graft placement decreased, autogenous fistula formation increased and the rates of complications detected on the fistula decreased [4-9]. Early failures may be detected by implementing a routine follow-up program after the fistula procedure and required interventions may be performed timely. Furthermore, this method may be used for diagnosis of possible complications such as thrombus, pseudoaneurysm steal syndrome and venous hypertension.

The aim of the present prospective, randomized controlled study was to search the effect of preoperative evaluation of the AVF performed on the patients with ESRF by DUS on AVF patency.

\section{METHODS}

\section{Patients}

One hundred and three patients who referred to our clinic for AVF procedure was evaluated. A detailed informed consent form was signed by all the participants. The present study was carried out in accordance with Helsinki standards and good clinical practice standards. The group whom AVF would be created according to physical examination findings only was defined as the control group or DUS (-) group whereas the group whom limb assessment was performed by preoperative DUS was determined as the study group or DUS (+) group. The physical examination criteria on the limb that AVF would be created for hemodialysis were presented in Table 1.
Twenty patients who met the physical examination criteria were included into the control group. Only one upper extremity was used in each patient. Non dominant arm was preferred for fistula formation.

The patients with a radial artery and cephalic vein diameters below $1.5 \mathrm{~cm}$; any stenosis, sclerosis, thrombus and occlusion on the cephalic vein and subclavian vein; lack of triphasic flow form on the radial artery and a significant stenosis in the radial artery all which were detected by preoperative DUS were excluded from the study. Twenty patients who met the DUS criteria were included into the control group.

Functionality of the AVFs created in both groups, presence of any thrill and whether the patient was taken into hemodialysis from the fistula created were controlled for 12 months. All the patients in both groups completed the study.

\section{Preoperative DUS Assessment}

In the present study, the requirement that the diameters of the radial artery and cephalic vein should be larger than $1.5 \mathrm{~mm}$ for the AVF created was determined. The patients were positioned at supine position following DUS; and the arms were fixed at 60 degrees on a comfortable position adjacent to the body to limit potential changes of some measurement such as internal lumen diameter and flow angle. A high-resolution DUS (Sonosite Micromaxx Ultrasound System model,USA) with a 10 to $12 \mathrm{MHz}$ linear alignment probe was used to take records. Each DUS scan lasted about 30 minutes. Colored Doppler and gray scale examinations were used for evaluation of spaces and anatomic variations of the radial artery

Table 1. Physical examination criteria for AVF

\begin{tabular}{l}
\hline $1 . \quad$ The visible vein length should be longer than $5 \mathrm{~cm}$ and may be compressed by superficial \\
palpation. \\
$2 . \quad$ The palpable strength of the arterial pulse should be over 2 (at a subjective scale determined \\
between 0 and $2 ; 0:$ no pulse; 1 : weak pulse; $2:$ normal pulse) [10]. \\
$3 . \quad$ No catheter should be inserted from the limb where the fistula procedure is planned. \\
$4 . \quad$ No previous fistula procedure should be performed from the limb which was planned for fistula \\
procedure \\
$5 . \quad$ No venous collaterals should exist on the shoulder area \\
$6 . \quad$ No lymphedema should exist \\
$7 . \quad$ Allen test should be negative \\
8. \\
No history of thrombophlebitis on the upper limb \\
N. No history of surgical procedure on the upper limb
\end{tabular}


Table 2. Demographic features of the patients

\begin{tabular}{lccc}
\hline & $\begin{array}{c}\text { Control Group } \\
\text { DUS (-) } \\
(\mathbf{n}=\mathbf{2 0})\end{array}$ & $\begin{array}{c}\text { Study Group } \\
\text { DUS (+) } \\
(\mathbf{n}=\mathbf{2 0})\end{array}$ & $\boldsymbol{p}$ value \\
\hline Age(years) & $56.10 \pm 12.35$ & $57.25 \pm 13.34$ & $>0.05^{*}$ \\
Male gender & $9(45 \%)$ & $10(50 \%)$ & $0.750^{\#}$ \\
Hypertension & $3(15 \%)$ & $6(30 \%)$ & $0.451^{\mathrm{a}}$ \\
Diabetes mellitus & $5(25 \%)$ & $6(30 \%)$ & $0.720^{\#}$ \\
\hline
\end{tabular}

DUS = Doppler ultrasonography. ${ }^{*}$ Student's t test, ${ }^{\#}$ Pearson Chi- Suquare test, ${ }^{a}$ Fisher's Exact test

and cephalic vein bilaterally, of which suitability for AVF was searched. When stenosis was suspected via colored DUS, waveforms were reviewed through the gray scale. The diameter of the radial artery at anteroposterior dimension and transverse plane through gray-scale ultrasonography on the wrist (1a). Diameter of the cephalic vein was measured individually on the wrist, elbow and mid-arm (Figure 1b). Venous measurements were also repeated by a tourniquet placed on the middle of the arm (Figure 2a). All cephalic vein and subclavian vein were examined. Sclerosis, stenosis and occlusion states were recorded. Any thrombus was controlled by slightly compressing on each vein (Figure $2 b$ ).

\section{Surgical Technique}

Brescio-Cimino procedure was implemented by preferring the non-dominant arm if both limbs are suitable. A parallel incision was done onto the forearm on the wrist area and the vessels were dissected from the surrounding tissues, liberated and hung by a ruler. The patients were heparinized (5,000 IU) before vascular occlusion; an approximately 5-7 $\mathrm{mm}$ longitudinal arteriotomy and venotomy were performed on the revealed vessels. Proximal end of the vein was closed; and end to side arteroivenous anastomosis was performed by 7/0 polypropylen suture.

\section{Postoperative Assessment of Arteriovenous Fistula}

Detection of murmur by auscultation and existence of thrill by palpation were assessed as a successful AVF procedure just after the procedure and at day 1 . Thrill was controlled in the patients during the control visits at day 10, months 1, 3,6 and 12. The patients were taken into hemodialysis sessions at day 2. A successful AVF procedure was considered as implementation of a 4-hour hemodialysis through a 16

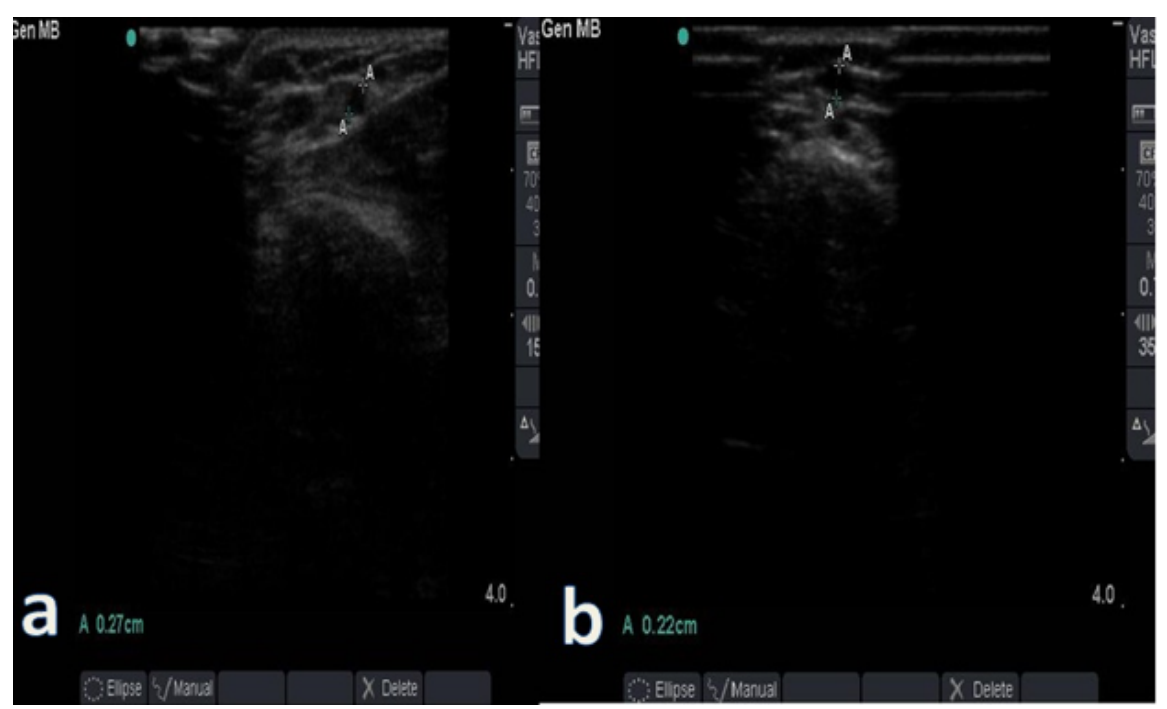

Figure 1. Measurement diameter of vessels by DUS. Radial artery (a) and cephalic vein (b). DUS = Doppler ultrasonography 


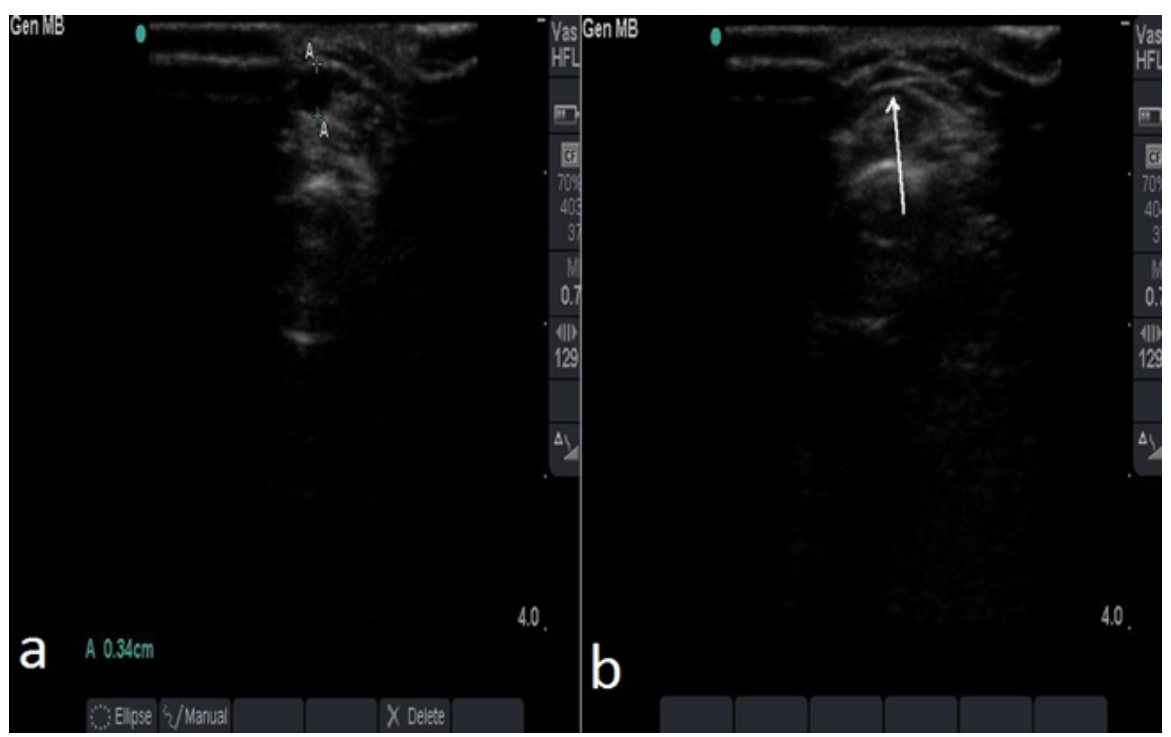

Figure 2. Cephalic vein image; (a) measurement diameter of cephalic vein with upper arm compression, (b) demonstration of cephalic vein compression with DUS (arrow). DUS = Doppler ultrasonography

gauge branule at $400 \mathrm{ml} / \mathrm{min}$ blood flow rate, three times a week. Primary patency of AVF was determined as the time interval between fistula creation day and the day that trreatment is required because of dysfunction.

\section{Statistical Analysis}

In such single-sided design with a significance level of 0.05 , the total number of the patients required for the sample size was 40; and these were divided into two equal parts as the control and the study groups including 20 patients each. We concluded that 40 patients would be sufficient for this study. Chi-square analysis was used for evaluation of categorical variables. Primary patency was analysed through Kaplan Mayer method. The t-test or corresponding non-parametric test was used for analysis of continuous variables. A $p<0.05$ value was accepted as statistically significant for all analyses. The SPSS 15.0 program was used for statistical evaluation.

\section{RESULTS}

No Twenty patients in the DUS $(+)$ group $(50 \%$ male, mean age: $57.25 \pm 13.34$ years) and 20 patients in the DUS (-) group (45\% male, mean age: $56.10 \pm$ 12.35) were recorded in the study. Demographic and clinical properties of the subjects were summarized in
Table 2. Both DUS (+) group and DUS (-) group were similar in terms of demographic properties.

Kaplan-Meier curves; cumulative primary patency rates DUS (+) group at days 1, 10, months 1, 3, 6 and 12 were $100 \%, 100 \%, 100 \%, 100 \%, 95 \%$ and $95 \%$; respectively. DUS (-) groups at days 1, 10, months 1 , 3,6 and 12 were were $80 \%, 75 \%, 75 \%, 70 \%, 65 \%$ and $65 \%$; respectively. Cumulative primary patency rates between DUS (+) group and DUS (-) group for 12 months were $95 \%$ and $65 \%$, respectively. In the DUS $(+)$ group, primary patency rates were superiority during follow-up periods.

There were 7 patients with AVF failure whom thrill could not be palpated during follow-up period in DUS (-) group. DUS was performed to reveal causes of AVF failure in these patients. Four unsuccessful AVFs were detected at postoperative day one. Chronic thrombotic modifications were detected in 3 patients whereas 1 patient presented an anastomosis defect. An insufficient radial artery flow existed in the unsuccessful fistula at postoperative day 10. A hyperplasia of the intima which causes stenosis in the juxta-anastomosis zone was detected in 2 fistulas which were considered as failure during the followups at months 3 and 6 . In the DUS (+) group, intimal hyperplasia was detected on the anastomosis line in 1 fistula which was considered as failure at month 6 .

None of the patients presented hemorrhage, edema, minor neural damage and wound site infection. 


\section{DISCUSSION}

This iA detailed physical examination is essential for a patient with chronic renal failure whom vascular procedure is planned. However, a preoperative radiological assessment of arterial and venous formations of the limb of which a vascular procedure is planned would have a positive contribution on the surgical plan [3-8].

Patency of Brescio-Cimino fistula is around 80\% despite developments of surgical techniques, training of the hemodialysis staff and novel medical methods [2]. A wide range of the patients with AVF, different surgical techniques and different anatomic sites for AVF make the randomization difficult. Same problem exists in the studies performed with DUS. Therefore, we assessed the patients whose overall status is well, who did not have any fistula procedure and catheter insertion.

Many studies focused on use of preoperative DUS before AVF creation $[5-8,11,12]$. An analysis showed that AVF remains unsuccesful during short-term when subclavian vein flow rate is below $400 \mathrm{ml} / \mathrm{min}$ detected by preoperative DUS. Some authors associated the arterial flow rate over $400 \mathrm{ml} / \mathrm{min}$ with high fistula flow rate; another study reported that an arterial or venous diameter below $1.6 \mathrm{~mm}$ is a reliable indicator for a possible AVF failure. However, increase

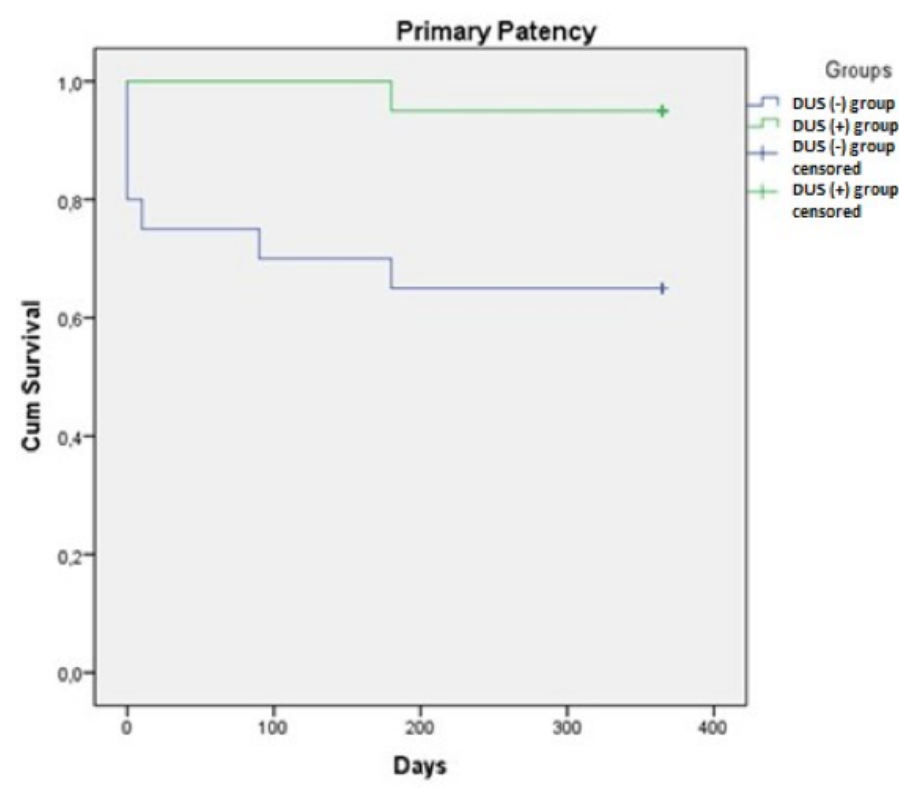

Figure 3. Kaplan-Meier curves; cumulative primary patency rates for twelve months. DUS = Doppler ultrasonography in AVF patency as well as decrease in AVF failure within short-term were reported as preoperative DUS using criteria in case of minimal arterial diameter of 2 $\mathrm{mm}$ and minimal venous diameter of $2.5 \mathrm{~mm}$ [13].

A similar and interesting study demonstrated that preoperative use of the DUS increase the achievement rate in AVF surgery and the surgical procedure was modified in $31 \%$ of 52 patients in terms of AVF site. The findings of the present study support use of preoperative DUS for AVF procedure. A tourniquet placed on the proximal side may enable measurement of maximum or real diameters of venous formations for evaluation of venous diameters. The venous congestion pressure should be $>40 \mathrm{mmHg}$ for an ideal measurement. In addditon to the venous diameter, a non-defective venous length above $10 \mathrm{~cm}$ detected by DUS is determinative at AVF function [14]. Venous diameters were measured without and with a tourniquet placed on the mid-arm and inflated by 40 mm Hg (Figure 2a).

A previous study assessed AVF patency at 3month following mapping by preoperative DUS and detected a direct proportion between venous diameter and patency. The veins with a diameter between 1.5 and $3.9 \mathrm{~mm}$ revealed an average patency rate of $71.08 \%$ at one-year whereas patency of smaller veins (1.5 to $2 \mathrm{~mm}$ ) was detected as $20 \%$ [15]. In another study, upon dysfunction of the fistula created on the forearm following venous mapping on the forearm and arm by preoperative DUS, same mapping process was used and a fistula was opened on the arm and a total achievement by $95 \%$ was reached [16]. It was reported in a study that preoperative assessment would be useful before AVF procedure; however, universal parameters were not determined yet [14]. In line with the literature, primary patency rates were found significantly higher in the AVF created through preoperative coloured DUS in the DUS $(+)$ group and such higher rates were statistically significant $(p=$ 0.022) (Figure 3). There was not any difference detected in terms of thrill palpation between the DUS $(+)$ and DUS (-) groups just after the AVF procedure and early postoperative period (early success of AVF). However, regular dialysis, AVF patency rates were found significantly higher in the DUS (+) group.

The difference of the present study was carrying out the study on the most eligible patient group for AVF procedure. These patients who had AVF 
procedure first were selected by the aforesaid exclusion criteria and only 40 of 103 patients met the inclusion criteria. A statistically significant higher patency rates in the DUS (+) group than the DUS (-) group during 12-month follow-up period reveals that such easily applicable imaging method, the DUS should be used routinely before AVF procedure.

\section{The Limitations of the Study}

Our study has one limitation. The small sample size may be to considered as the one limitation of this study, but the excess of exclusion criteria has minimized the risk factors for patency. Further prospective studies with a larger number of patients are required.

\section{CONCLUSION}

In Consequently, although the operative strategy is usually considered sufficient according to the physical examination of the surgeon before creation of AVF in practice, the DUS performed before AVF procedure would increase the primary patency rates of AVF created between the most convenient vessels and reduce the procedure failure. Accurate patient selection, accurate patient preparation seems to be a preliminary condition for high rate of primary patency and we believe that DUS assessment is valid and necessary.

\section{Authorship contributions}

Consept-Design: NK, KKÖ; Data collection : NK, KKÖ; Analysis: KKÖ, NK; Literature search: KKÖ, NK; Writing: KKÖ, NK; Critical review:NK, KKÖ.

\section{Conflict of interest}

The authors disclosed no conflict of interest during the preparation or publication of this manuscript.

\section{Financing}

The authors disclosed that they did not receive any grant during conduction or writing of this study.

\section{REFERENCES}

[1] Rooijens PP, Burgmans JP, Yo TI, Hop WC, de Smet AA, van den Dorpel MA, et al. Autogenous radial-cephalic or prosthetic brachialantecubital forearm loop AVF in patients with compromised vessels? A randomized, multicenter study of the patency of primary hemodialysis access. J Vasc Surg 2005;42:481-6.

[2] Rahman A, Özsin KK. Upper extremity otogen arteriovenous fistulas for hemodialysis. Turkish J Vasc Surg 2007;16:19-24.

[3] Brescia MI, Cimino JC, Appel K. Chronic hemodialysis using venipuncture and surgically created arteriovenous fistulas. N Eng J Med 1966;275:1089-92.

[4] Guedes Marques M, Ibeas J, Botelho C, Maia P, Ponce P. Doppler ultrasound: a powerful tool for vascular access surveillance. Semin Dial 2015;28:206-210.

[5] Wong CS, McNicholas N, Healy D, Clarke-Moloney M, Coffey JC, Grace PA, et al. A systematic review of preoperative duplex ultrasonography and arteriovenous fistula formation. J Vasc Surg 2013;57:1129-33.

[6] Ko SH, Bandyk DF, Hodgkiss-Harlow KD, Barleben A, Lane J 3rd. Estimation of brachial artery volume flow by duplex ultrasound imaging predicts dialysis access maturation. J Vasc Surg 2015;61:1521-7.

[7] Lomonte C, Meola M, Petrucci I, Casucci F, Basile C. The key role of color Doppler ultrasound in the work-up of hemodialysis vascular access. Semin Dial 2015;28:211-5.

[8] Matsui S, Nakai K, Taniguchi T, Nagai T, Yokomatsu T, Kono Y, et al. Systematic evaluation of vascular access by color-Doppler ultrasound decreased the incidence of emergent vascular access intervention therapy and X-ray exposure time: a single-center observational study. Ther Apher Dial 2012;16:169-72.

[9] Older RA, Gizienski TA, Wilkowski MJ, Angle JF, Cote DA. Hemodialysis access stenosis: early detection with color DUS. Radiology 1998;207:161-4.

[10] Sidawy AN1, Spergel LM, Besarab A, Allon M, Jennings WC, Padberg FT Jr, et al. The Society for Vascular Surgery: clinical practice guidelines for the surgical placement and maintenance of arteriovenous hemodialysis access. J Vasc Surg 2008;48:2S-25S.

[11] Bay WH, Henry ML, Lazarus JM, Lew NL, Ling J, Lowrie EG. Predicting hemodialysis access failure with color flow Doppler ultrasound. Am J Nephrol 1998;18:296-304.

[12] Dossabhoy NR, Ram SJ, Nassar R, Work J, Eason JM, Paulson WD. Stenosis surveillance of hemodialysis grafts by duplex ultrasound reduces hospitalizations and cost of care. Semin Dial 2005;18:550-7.

[13] Schanzer H, Skladany M. Vascular access for dialysis. In: Haimovici H. Editor. Haimovici's Vascular Surgery: Principles and Techniques. 4th ed. Cambridge: Black Science, 1996. pp.1028-41.

[14] Planken RN, Tordoir JHM, Duijm LEM, de Haan MW, Leiner T. Current techniques for assessment of upper extremity vasculature prior to hemodialysis vascular access creation. Eur Radiol 2007:17:3001-11. [15] Hamish M, Geddoa E, Reda A, Kambal A, Zarka A, Altayar A, et al. Relationship between vessel size and vascular access patency based on preoperatively ultrasound Doppler. Int Surg 2008;93:6-14.

[16] Nguyen VD, Treat L, Griffith C, Robinson K. Creation of secondary AV fistulas from failed hemodialysis grafts: the role of routine vein mapping. J Vasc Access 2007;8:91-6. 